\title{
Modelling Unsaturated Soil Response Beyond Residual Suction State via Vapor-Pressure Controlled Triaxial Testing
}

\author{
Mathilde Morvan ${ }^{1}$, Ujwalkumar D. Patil $2^{2 *}$, Laureano R. Hoyos ${ }^{3}$, Surya S. C. Congress ${ }^{4}$, and Anand J. Puppala ${ }^{4}$ \\ ${ }^{1}$ Department of Civil Engineering, Polytech Clermont-Ferrand, Université Clermont Auvergne, Institut Pascal, F-63171 Aubière, France \\ ${ }^{2}$ Department of Civil Engineering, School of Engineering, University of Guam, Mangilao, Guam 96923, Guam \\ ${ }^{3}$ Department of Civil Engineering, University of Texas at Arlington, Arlington, Texas 76019, USA \\ ${ }^{4}$ Department of Civil Engineering, Texas A \& M University, College Station 77843, Texas, USA
}

\begin{abstract}
Most of the previous research has been focused on developing and validating constitutive models to predict response of unsaturated soils in low-medium suction range. However, there is a scarcity of efforts in developing soil models to simulate its mechanical response in high suction range, particularly above the residual suction. This article presents a new constitutive model introducing net stress and suction as two independent variables. Furthermore, non-associative flow rule incorporating modified stressdilatancy relationship to take unsaturated state into account is introduced to improve the model results in low-medium to high suction range. The essential soil model parameters are calibrated using suctioncontrolled triaxial test results for predictions of compacted silty sand response at high values of total suction above residual suction. Preliminary simulations show that proposed model can reasonably simulate the postpeak strain softening response obtained from suction-controlled CTC tests above residual suction value with reasonable accuracy. Although, the proposed model captures initial compression followed by dilation volumetric response with reasonable accuracy, it needs some improvements to be able to capture volumetric response accurately over entire suction range.
\end{abstract}

\section{Introduction}

The original form of Barcelona Basic Model (BBM) proposed by Alonso et al. [1] is limited in application as it is unable to capture the behaviour of sands, including the post peak strain softening and the transition of initial compressive volumetric strains changes followed by dilation volume changes that can occur in sands. Bardet [2] was the first among others to propose a comprehensive soil model based on concept of Bounding Surface (BS) plasticity to reproduce post-peak softening and dilatational response of dry and saturated sands. Since its inception, the Bounding Surface (BS) plasticity theory has been continuously been used and modified by past researchers over last four decades. Based on the concept of BS plasticity theory, several researchers have attempted in developing constitutive models for saturated and dry soils $[3,4,5,6]$ and for unsaturated soils $[7,8,9,10]$.

Russell and Khalili [7], extended the BS plasticity theory to unsaturated soils in a critical state framework using concept of effective stress taking into account the particle crushing associated with shearing. Morvan et al. [8], further presented an extension of Bardet [2] constitutive model based on the concept of BS plasticity under a critical state framework for unsaturated soils and tested it successfully on data obtained from suctioncontrolled CD triaxial tests conducted on dense Kurnell sand by Russell [11]. This was further fine-tuned and calibrated to mimic the stress strain and volume change response obtained from suction-controlled consolidated drained (CD) triaxial test results on compacted silty sand over the range of $50-750 \mathrm{kPa}$ matric suction by Patil et al. [12].

However, the constitutive model previously developed by Patil et al. [10] is limited and incapable of reproducing the behaviour of unsaturated soils in high suction range, especially beyond the residual suction. Although the plastic driver of this model based on Bardet [2] is of interest, the concept of enlarged effective stress does not seem to apply for high suction.

This article presents the modifications made to introduce net stress and suction as two independent variables. Furthermore, non-associative flow rule incorporating stress-dilatancy by Rowe [13] and Bolton [14] is introduced to improve the model results. Stressdilatancy relationship was chosen in accordance to $\mathrm{Yu}$ [6] and further modified to take unsaturated state into account.

The proposed BS model is first calibrated using comprehensive suction-controlled consolidated drained triaxial test data obtained between air entry value and residual suction (i.e., suction between $50-750 \mathrm{kPa}$ ) and then beyond residual suction (i.e., between 20,000 $300,000 \mathrm{kPa}$ ) under net confinement of $100-300 \mathrm{kPa}$ of silty sand $[12,15]$ to determine the calibrated parameters

Corresponding author: patilu@ triton.uog.edu 
which are further used to reproduce the triaxial behaviour of unsaturated silty sand. Finally, the results of modified model are compared with the experimental data of triaxial tests for low and high suction ranges.

\section{Materials and Equipment}

Poorly graded silty sand (SM) was used to produce triaxial test specimen of 2.8-inch diameter and 5.6-inch height with over consolidated stress history. The specimens were statically compacted using triaxial frame to a target density of $1.87 \mathrm{~g} / \mathrm{cm}^{3}$ and optimum water content of $12.2 \%$.

Suction-controlled consolidated drained triaxial tests were conducted on identical silty sand specimens using a double-walled triaxial test equipment via axis-translation technique (suction $=50-750 \mathrm{kPa}$ ) and via relativehumidity technique (suction $=20-300 \mathrm{MPa}$ ) under net confinement of 100-300 kPa. Detailed test protocol and results can be further obtained in Patil et al., [12, 15]. Figure 1 illustrates the modified triaxial test equipment used in this experimental program.

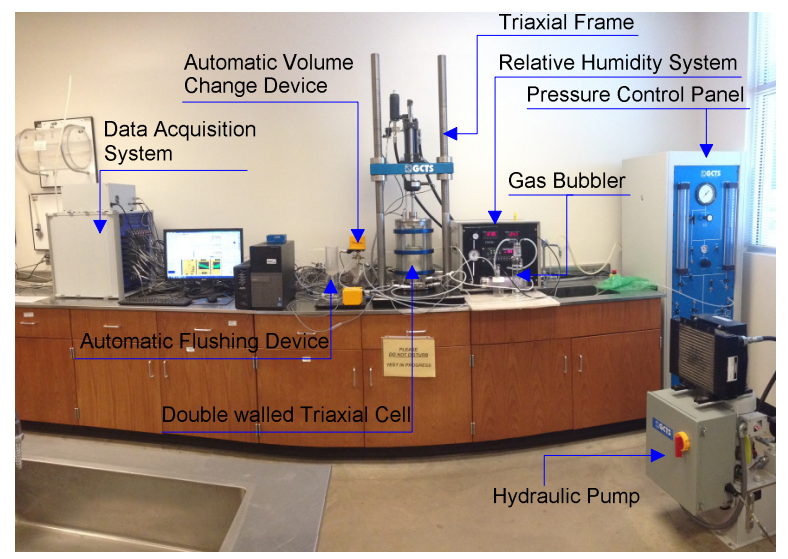

Fig. 1. Panoramic view of modified Triaxial test equipment to accommodate relative humidity technique to test soil at high suction

\section{Bounding surface-based model for unsaturated soils with two independent variables (BSMU2)}

In the proposed model, Bardet's [2] plastic driver is used. Modifications are made to introduce net stress and suction as two independent variables. Hence this model is named as bounding surface-based model with two independent variables (BSMU2). It is based on the concept of bounding surface plasticity that allow a smooth transition between elastic and plastic domains and also is capable of capturing the post-peak behaviour and softening of the soil $[7,16]$.

We work in the cylindrical symmetry and adopt the classic notation of triaxial variables $(p, q)$, where $p$ is the mean net stress and $q$ is the deviatoric stress and $\eta$ is the stress ratio. To summarize:

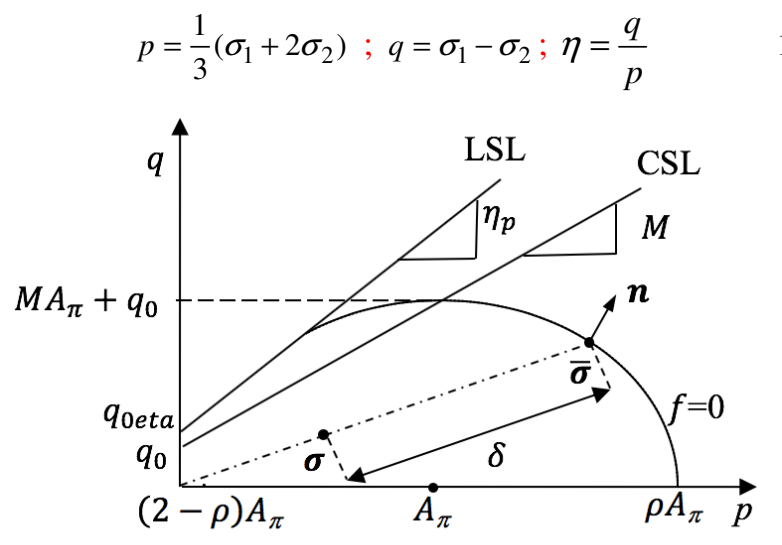

Fig. 2. Radial mapping algorithm in BSMU2 model.

The conjugate strains are the volumetric $\varepsilon_{p}$ and deviatoric $\varepsilon_{q}$ strains, defined by:

$$
\varepsilon_{p}=\varepsilon_{1}+2 \varepsilon_{2} ; \varepsilon_{q}=\frac{2}{3}\left(\varepsilon_{1}-\varepsilon_{2}\right)
$$

We assume that all strain components can be decomposed into an elastic and a plastic part:

$$
\varepsilon_{p}=\varepsilon_{p}^{e}+\varepsilon_{p}^{p} ; \varepsilon_{q}=\varepsilon_{q}^{e}+\varepsilon_{q}^{p}
$$

Bardet's model has been adapted to unsaturated soils. The original bounding surface is an ellipse (Fig. 2) and its original equation is given by:

$$
f\left(\bar{p}, \bar{q}, \varepsilon_{p}^{p}\right)=\left(\frac{\bar{p}-A}{\rho-1}\right)^{2}+\left(\frac{\bar{q}}{M}\right)^{2}-(A)^{2}
$$

Where $\bar{p}$ is the mean net stress and $\bar{\sigma}(\bar{p}, \bar{q})$ is the image point of the current stress state $\sigma(p, q)$ on the bounding surface (Fig.2).

Few modifications were necessary to incorporated two independent variables needed to describe unsaturated state. The slope $\eta_{p}$ of Limit State Line (LSL) that limits the reachable stress domain and the slope $M$ of Critical State Line (CSL) is considered constant but $\mathrm{Y}$-intercept depends on suction. A logarithmic law is considered to take high suction into account:

$$
\begin{gathered}
q_{0}=a_{q} \ln (s)+b_{q} \\
q_{0 \text { eta }}=a_{\text {qeta }} \ln (s)+b_{\text {qeta }}
\end{gathered}
$$

Where $q_{0}$ is the Y-intercept of CSL and $q_{\text {oeta }}$ the Yintercept of LSL, $s$ the suction and $a_{q} a_{\text {qeta }}, b_{q}, b_{\text {qeta }}$ some parameters. These four parameters were obtained by studying the evolution of CSL and LSL with suction.

The critical state variable $\lambda$ dependency toward suction is taken similar to BBM model [1]:

$$
\lambda=\lambda_{0} \cdot((1-r) \exp (-\beta s)+r)
$$

where $\lambda_{0}$ is the saturated critical state variable [17] and $\beta$ and $r$ are parameters defining suction dependency of $\lambda$. 
The bounding surface has to be modified by taking $A$ variation into account but also the evolution of $\mathrm{Y}$ intercept of CSL (Fig. 2).

$f\left(\bar{p}, \bar{q}, \varepsilon_{p}^{p}, s\right)=\left(\frac{\bar{p}-A_{\pi}}{\rho-1}\right)^{2}+\left(\frac{\bar{q}}{M+q_{0} / A_{\pi}}\right)^{2}-\left(A_{\pi}\right)^{2}$

Where:

$$
A_{\pi}=p_{c}\left(\frac{A_{0}}{p_{c}}\right)^{\frac{\lambda_{0}-\kappa}{\lambda-\kappa}}
$$

The image points are obtained by radial mapping (Fig.2):

$$
\bar{p}=\gamma A_{\pi} ; \bar{q}=\gamma x M A_{\pi} ; x=\frac{q}{M p+q_{0}}
$$

with :

$$
\gamma=\frac{1+(\rho-1) \sqrt{1+x^{2} \rho(\rho-2)}}{1+(\rho-1)^{2} x^{2}}
$$

The definition of the plastic modulus and of the plastic multiplier used with the consistency relation give us:

$$
\frac{\partial f}{\partial \varepsilon_{p}^{p}} \frac{d \varepsilon_{p}^{p}}{\left\|\frac{\partial f}{\partial \bar{\sigma}}\right\|}=-H_{b} d \varsigma
$$

The gradient of $f$ is composed of three terms:

$$
\frac{\partial f}{\partial \bar{\sigma}}=\left(\frac{\partial f}{\partial \bar{p}} ; \frac{\partial f}{\partial \bar{q}} ; \frac{\partial f}{\partial s}\right)^{t}
$$

After few calculations, the normalized components of this gradient of $f$ are given by:

$$
\begin{gathered}
n_{p}=\frac{\frac{\partial f}{\partial \overline{\bar{p}}}}{\left\|\frac{\partial f}{\partial \bar{\sigma}}\right\|} \\
n_{q}=\frac{\frac{\partial f}{\partial \bar{q}}}{\left\|\frac{\partial f}{\partial \bar{\sigma}}\right\|} \\
n_{s}=\frac{\frac{\partial f}{\partial s}}{\left\|\frac{\partial f}{\partial \bar{\sigma}}\right\|}
\end{gathered}
$$

where:

$$
g=\left\|\frac{\partial f}{\partial \bar{\sigma}}\right\| \frac{M(\rho-1)^{2}}{2 A_{\pi}}
$$

The plastic modulus can then be calculated:

$$
H_{b}=-\frac{\partial f}{\partial A_{\pi}} \frac{\partial A_{\pi}}{\partial \varepsilon_{p}^{p}} \frac{\partial f}{\partial p}\left(\frac{2 A_{\pi}}{\left\|\frac{\partial f}{\partial \bar{\sigma}}\right\| M(\rho-1)^{2}}\right)^{2}
$$

When the stress point lies inside the bounding surface, a second term has to be added to the plastic modulus:

$$
H=H_{b}+H_{f}
$$

We can take:

$$
H_{f}=\frac{1+e_{0}}{\lambda(s)-\kappa} \frac{\delta}{\delta_{\max }-\delta} h_{0} \frac{\left\langle\eta_{p} p+q_{0 e t a}-q\right\rangle}{M}
$$

Where $\eta_{p}$ is the slope of the Limit State Line (Fig.2). Non-associative flow rule incorporating stress-dilatancy by Rowe [13] and Bolton [14] is introduced to improve the model results. Stress-dilatancy relationship was chosen in accordance to $\mathrm{Yu}$ [6] and further modified to take unsaturated state into account. The plastic potential $\mathrm{g}$ is based on the following stress dilatancy relation by $\mathrm{Yu}[6]$ :

$$
\frac{d \varepsilon_{p}^{p}}{d \varepsilon_{q}^{p}}=\frac{M^{n}-m \eta^{n}}{m \eta^{n-1}}
$$

As for $\mathrm{f}$ we can obtain normal vector to the plastic potential, and we get for example:

$$
m_{p}=\frac{\frac{\partial g}{\partial p}}{\left\|\frac{\partial g}{\partial \sigma}\right\|}
$$

Plastic strains are given by:

$$
\begin{array}{r}
d \varepsilon_{p}^{p}=\left\langle\frac{1}{H}\left(n_{p} d p+n_{q} d q+n_{s} d s\right)\right\rangle m_{p} \\
d \varepsilon_{q}^{p}=\left\langle\frac{1}{H}\left(n_{p} d p+n_{q} d q+n_{s} d s\right)\right\rangle m_{q}
\end{array}
$$

Elastic strains are defined by classical relation:

$$
d \varepsilon_{p}^{e}=\frac{\kappa}{\left(1+e_{0}\right)} \frac{d p}{p} ; d \varepsilon_{q}^{e}=\frac{2(1+v)}{9(1-2 v)} \frac{\kappa}{1+e_{0}} \frac{d q}{p}
$$

In total this model needs 16 parameters (see Table 1) to describe mechanical behaviour of saturated soils and unsaturated soils for low, medium and high suction.

\section{Experimental Test Results, Model Calibration and Simulations}

The saturated soil test results from triaxial testing program for silty sand showed strain-hardening type of response while the volume change response was compressive type (Fig. 3). On the other hand, the stress strain response turned to becoming strain-softening type and the peak stress becomes prominent with increasing suction (Fig. 4 and 5). This is the challenge for soil modelers as the traditional BBM is unable to capture the post-peak softening and dilational volume change. Previously, Morvan et al. [8] proposed a BS model and later Patil et al. [10] modified it to capture above soil 
response between low to medium suction range. However, it failed to reproduce the triaxial response at high suction, especially beyond residual suction. This research paper focusses on reproducing such unsaturated soil response through modifications to soil model proposed by Morvan et al. [8] and Patil et al. [10].

This model has been modified to incorporate effects of partial saturation toward two independent variables: net stress and suction. The variation of the limit state line and critical state line was established using triaxial tests at a confining pressure of $100 \mathrm{kPa}$. Model parameters were chosen in order to reproduce saturated soil behaviour. Saturated triaxial test results (Fig. 3) and isotropic compression tests were used to obtain model parameters $\kappa, \lambda_{0}, \rho, M, \eta_{p}, v, h_{0}, n . M, \eta_{p}$ are obtained by studying CSL and LSL for saturated state. Whereas $\rho, h_{o}$, and $n$ are adjusted using best fit method. Secondly, the remaining parameters were obtained using unsaturated triaxial tests at a confining pressure of $100 \mathrm{kPa}$. With unsaturated tests, it is possible to plot the evolution of CSL, LSL and $\lambda$ with suction and obtain $a_{q}, b_{q}, a_{\text {qeta }}, b_{\text {qeta }}, r, \beta$.

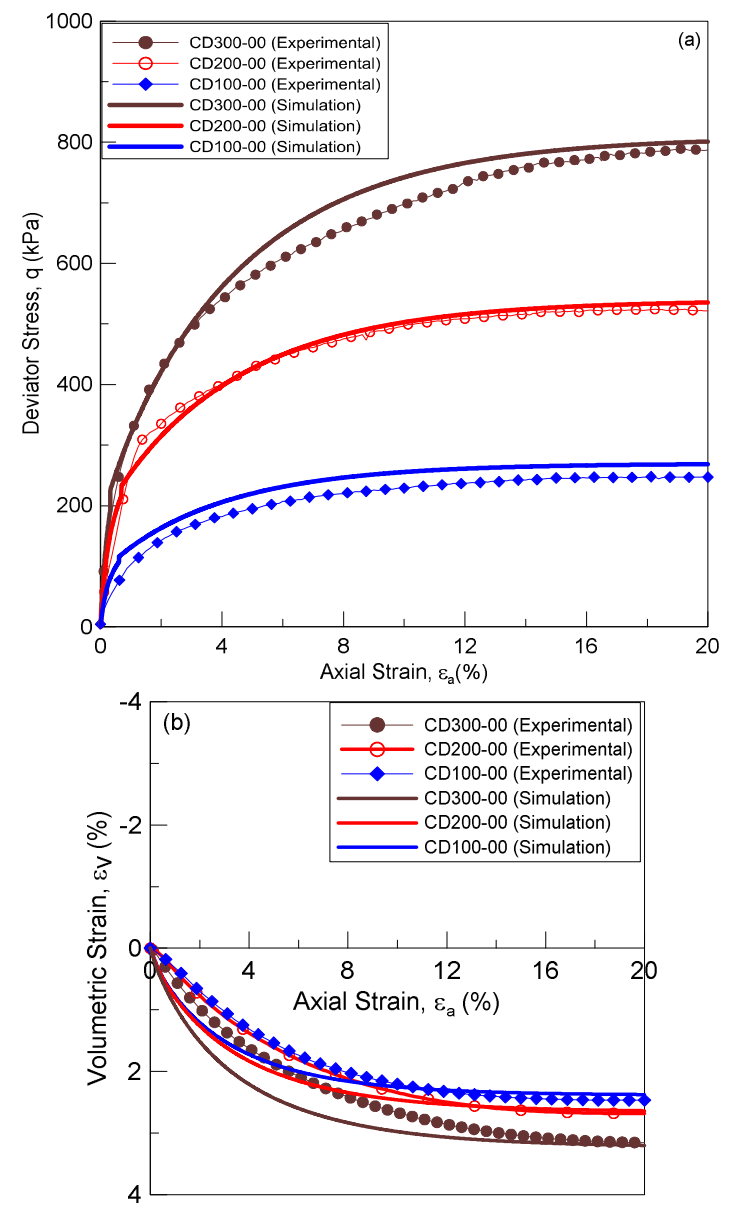

Fig 3. Experimental results of saturated compacted silty sand triaxial tests, (a) Stress-strain and (b) Volume change curves

The proposed model was programmed in Matlab platform using incremental calculation for each step of loading. Strain increment was imposed to generate stress-strain predictions. Parameters used for these simulations are given in Table 1.

Table 1. Parameters used for simulation

\begin{tabular}{|c|c|c|}
\hline Parameter & Unit & Value \\
\hline$\kappa$. & & 0.003 \\
\hline$v$ & & 0.3 \\
\hline$\lambda_{0}$ & & 0.04 \\
\hline$M$ & & 1.42 \\
\hline$\eta_{p}$ & & 1.8 \\
\hline$\rho$ & & 2.1 \\
\hline$h_{0}$ & & 5 \\
\hline$n$ & & 2 \\
\hline$r$ & & 0.8 \\
\hline$\beta$ & $\mathrm{kPa}-1$ & 0.002 \\
\hline$a_{q}$ & $\mathrm{kPa}$ & 34.6 \\
\hline$b_{q}$ & $\mathrm{kPa}$ & 67 \\
\hline$a_{q e t a}$ & $\mathrm{kPa}$ & 220 \\
\hline$b_{q e t a}$ & $\mathrm{kPa}$ & 95 \\
\hline$p_{c}$ & $\mathrm{kPa}$ & 46 \\
\hline$A_{0}$ & $\mathrm{kPa}$ & Varies \\
\hline & &
\end{tabular}
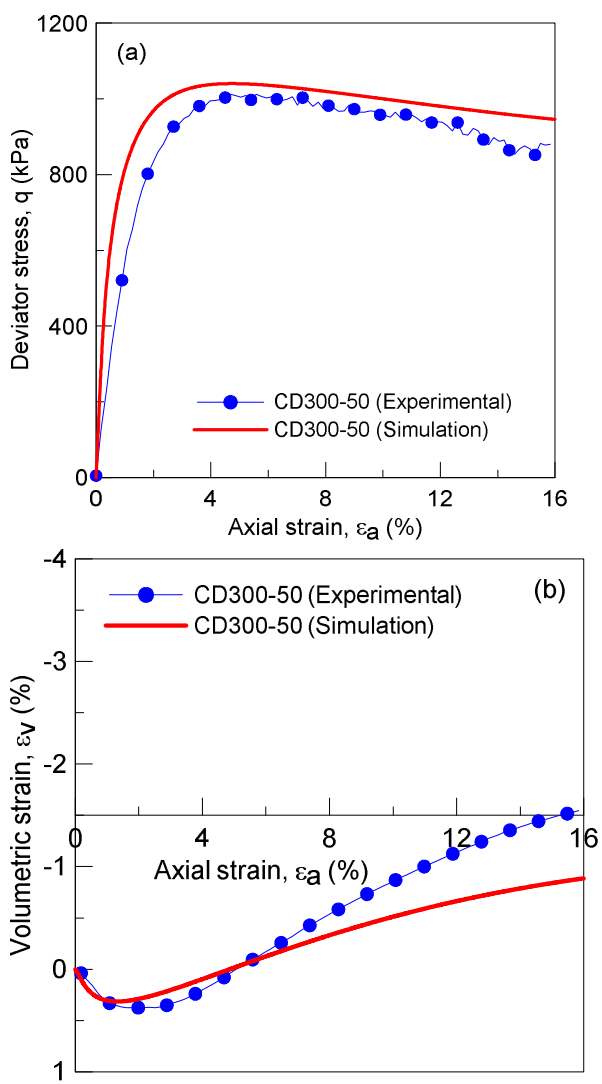

Fig 4. Experimental and BSMU2 Model predictions of compacted silty sand soil response from suction-controlled triaxial tests at $\mathrm{s}=50 \mathrm{kPa}$

Fig. 3 shows the experimental and simulations results for saturated soil $\mathrm{CD}$ triaxial testing. Clearly, the proposed BSMU2 model can reproduce the saturated stress-strain and volume change response. Results of suction-controlled $\mathrm{CD}$ triaxial tests at a confining 
pressure of $300 \mathrm{kPa}$ are illustrated in Figs 4 and 5. It can be seen that the experimental data and model predictions matches decently well which illustrates promising application of the proposed BSMU2 model at high suction. The proposed BSMU2 is able to smoothly capture the transition of strain-hardening type saturated response into unsaturated post-peak strain softening type. This illustrates the promise of proposed model. Although, the proposed model captures initial compression followed by dilation volumetric response with reasonable accuracy, volumetric response is not accurately predicted over entire range of suction. As suggested by the post peak behaviour for high suction a strain localisation may have occurred within the experimental test. This strain localisation may explain why the model simulation does not capture accurately the experimental data.
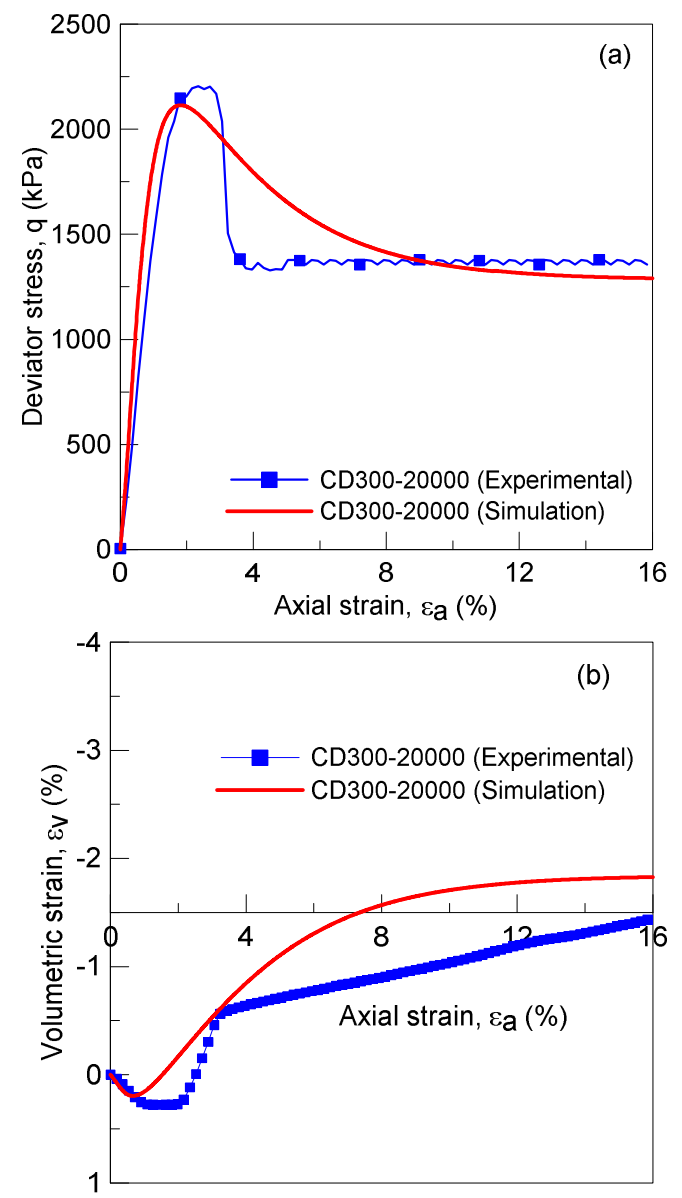

Fig 5. Experimental and BSMU2 Model predictions of compacted silty sand soil response from suction-controlled triaxial tests at $\mathrm{s}=20,000 \mathrm{kPa}$

\section{Conclusions}

Bardet's plastic driver is used to model unsaturated soils behaviour in high suction ranges. This plastic driver was previously used using enlarged effective stress (Patil et al. [10] to reproduce this unsaturated soil behaviour for low and medium suction range. This previously developed model was limited in application over low to medium suction range and was unable to mimic unsaturated soil response beyond residual suction (high suction). This model was further modified to incorporate effects of partial saturation toward two independent variables: net stress and suction.

The variation of the limit state line and critical state line was established using triaxial tests at a confining pressure of $100 \mathrm{kPa}$. Results of triaxial tests at a confining pressure of $300 \mathrm{kPa}$ are illustrated in this paper. Experimental data and model predictions compared decently well which illustrates its promising application at high suction. Although, the proposed model captures initial compression followed by dilation volumetric response with reasonable accuracy, volumetric response is not accurately predicted over entire range of suction. Strain localisation may explain why the model simulation does not capture accurately the experimental data.

\section{Acknowledgements}

The experimental work described in this paper is part of research project funded by National Science Foundation (NSFMRI award ID \# 1039956). This support is gratefully acknowledged. Any findings, conclusions, or recommendations expressed in this material are those of the authors and do not necessarily reflect the views of the National Science Foundation.

\section{References}

1. E.E. Alonso, A. Gens, A.A. Josa, Géotechnique, 40, 3, 405-430 (1990)

2. J.P. Bardet, Journal of engineering mechanics 112,11: 1198-1217 (1986)

3. Y.F. Dafalias, L.R. Herrmann, J. Eng. Mech., 112, 12, 1263-1291 (1986)

4. Z.L. Wang, Y.F. Dafalias, C.K. Shen, J. Eng. Mech. 116, 5, 983-1001 (1990)

5. H.I. Ling, H.B. Liu, Y. Mohri, T. Kawabata, J. Eng. Mech., 127, 9, 963-967 (2001)

6. H.S. Yu, Plasticity and Geotechnics (Springer, New York, 2006)

7. A.R. Russell, N. Khalili, International Journal for Numerical and Analytical Methods in Geomechanics, 30, 181-212 (2006).

8. M.M. Morvan, H. Wong, D. Branque, International Journal for Numerical and Analytical Methods in Geomechanics, 10 (34): 1512-1537 (2010)

9. J. Jiang, H.I. Ling, V.N. Kaliakin, J Appl Mech., 79, 3, 031010 (2012)

10. U.D. Patil, L.R. Hoyos, M.M. Morvan, A.J. Puppala, Int. J. Numer. Anal. Methods Geomech., 42, 14, 1741-1761 (2018)

11. A.R. Russel, Cavity expansion in unsaturated soils Ph.D. dissertation, The University of New South Wales, Australia (2004)

12. U.D. Patil, L.R. Hoyos, A.J. Puppala, Int. J. Geomech., 16, 6, D4016012 (2016). 
13. P.W. Rowe, Proc. Roy. Soc. A., 267, 500-527 (1962)

14. M.D. Bolton, Géotechnique, 36, No. I.65578 (1986).

15. U.D. Patil, A.J. Puppala, L.R. Hoyos, Geotechnical Testing Journal ASTM, 39, 5, 742-756 (2016)

16. Y.F. Dafalias, Journal of engineering mechanics, 112, 966-987 (1986)

17. A. Schofield and P. Wroth, Critical state soil mechanics (McGraw-Hill, New York, 1968). 